Fetal Diagn Ther 1997;12:370-372

\title{
Subject Index Vol. 12, 1997
}

Fetal Diagnosis Therapy

Abnormal karyotype 132 Abortions 140 Absent end diastolic flow 15 Accelerations, heart rate 236 Amniocentesis 283 -, early 140 Amnioinfusion 188 Amnionicity 363 Amnioreduction 50 Amniotic fluid 145,301 Aneuploidy 309 Antenatal diagnosis 306 Aqueous plastic extract 28 Ascites 43 Asplenia 266 Assessment, fetal 252 Atria 304 Attitudes 93

Bacterial vaginosis 244 Biliary atresia 306 Biochemical screening 153 Blood sampling, fetal 252 Bradycardia 236 Brain damage 286

- $\quad$ injury 68

Breech position 296

Calcium ATPase 270

Cardiac hemodynamics 332

Cataract 197

Catecholamine 314

Cephalocentesis 296

Cervical incompetence 274

Chemoreceptor 314

Choledochal cyst 306

Chorioamniotic membrane separation 81

Chorioangioma 61

Chorionic villus sampling 59, 140, 236

Chorionicity 363

Choroid plexus cysts 255

Chromosomal aberrations 255

Chromosome aberration 140

Cloacal dysgenesis 279

Cocaine 292

Color Doppler 360

- $\quad$ - echography 24

Computer analysis 319

Concordance 279

Congenital anomalies 216, 292

Conjoined twins 185

Cordocentesis 200, 252, 348

Core fragment 336 ß-Core 336 Corticosteroid(s) 170

- $\quad$ therapy 226

Creatine kinase 356

Cystic hygroma 132

Delivery 216 Differential diagnosis 306 Doppler imaging 61 
- $\quad$ velocimetry $85,332,353$

Double uterus 59

Down syndrome 93, 153, 156 Drug screening 248 Ductus arteriosus 46 Duodenal atresia 298 - duplication 298

Early amniocentesis 97

- pregnancy 132

Echocardiography 72,332

-, fetal 241

Echography 7

Ectopic pregnancy 336,340 Embryofetoscopy 136 Embryoscopy 136, 185 Emergency cervical cerclage 274 Endoscopy 188 Epidemiology 216 Erythromycin 89 Extrauterine fetal incubation 314 Extremity constriction 50

Femur shortening 156 Fetal abnormalities 340

ascites/hydrops 15

atrial flutter 46

behaviour 319

blood sampling 54

cardiac failure 241

circulation 178,314

death 286

demise 81

diastolic cardiac function 72

disease 232

distress 167

echocardiography 43

effects 97

endoscopy 76

eye 76

gender 301

goiter 54,200

KAHGER

Fax + 41613061234 E-Mail karger@karger.ch www. karger. com

(C) 1997 S. KargerAG, Basel

growth retardation 332

heart rate 170

- - monitoring 167

hemodynamics 72

hydrops 61,132

intrauterine transfusion 343

lamb 188

outcome 140

pulmonary circulation 226

rat 68

supraventricular tachycardia 205

surgery 81,145 
tachycardia 46

therapy $1,21,54,328$

thyrotoxicosis 54

urine 1

- production rate $343 \alpha$-Fetoprotein 85, 102, 301 Fetoscopy 136, 145 Fetus 248

First-trimester diagnosis 140 FISH 36, 309 Flecainide acetate 328 Flow cytometry 309 Flutter 205

Functional pulmonary atresia 43

Gastroschisis 7

Gender 304

Gene expression 270

Genetic diagnosis 255

Glycine 145

Graves' disease 54, 200

Growth 163

- $\quad$ retardation 210

Haematoma 163 Heart, human 270

- $\quad$ rate 236,319

Hepatic cyst 221

Hip and foot dislocations 140 Hispanic ethnicity 102 Histopathology 210

Hospitals, statistics and numerical data 2 Human chorionic gonadotropin 336 ß-Human chorionic gonadotropin 353 Humerus shortening 156 Hydrocephalus 296 Hydrops 205

- $\quad$ fetalis 40,241

Hyperthyroidism 24, 54

In utero surgery 188

Infarction 163

Interleukin-1 $\beta 7$

Interleukin-6 7

Intrauterine growth restriction 348

- retardation 170,226

infection 40

treatment 221

Intravascular transfusion 149 Isochromosome 20q 283

Laryngotracheoesophageal cleft 260

Laser coagulation, histology 32

Lateral ventricle 304

Light damage 76

Limb 97

Liver 232

Low unconjugated estriol 36

Lung abnormalities 360

Magnetic resonance imaging 360 Maternal hypoxemia 68

- $\quad$ serum screening 102

Maternal-fetal infection 66

Meconium 248 
Methylmalonic aciduria 21

Metronidazole 244

Mice 28

ß2-Microglobulin 1 Midtrimester 274 Monoamniotic twin 286 Monozygotic twins 279

Mosaicism 283 Movement 319

Multicystic encephalomalacia 286 Multiple gestation 59

Nd:YAG laser 32 Neonatal intensive care 216

mortality 216

outcomes 274 Neural tube defects 102 Newborn 216

Nonimmune hydrops fetalis 66 Nuchal translucency 270

Obstructive uropathy 1

$\mathrm{PaC0} 314$

Papanicolaou smear 244 ParvovirusB19 40,66 6 Perinatal mortality 216 Phagocyte 232 Placenta 163,210 Placental chorioangioma 241

vessel 32 Plastics 28 Pregnancy 226

complications 356

loss 236

outcome $85,216,353$

reduction 59

Prenatal diagnosis 1, 21, 24, 36, 46, 54, 66, 93,97, 132,136,156, 185,197,241,

$266,283,286,309,360$

effects 28

scan 260

ultrasonography 298

Fetal Diagn Ther Vol. 12, 1997

371

Preterm birth 244

- $\quad$ labor 89

Proliferating cell nuclear antigen 210 Pulmonary pulsatility index 226

- $\quad$ resistance index 226

Quadruplets 363

Randomized controlled trial 97 Red cell alloimmunization 149 Renal dysplasia 1 Respiratory problems 97 Rhesus isoimmunization 343 Right isomerism 266 Robert's syndrome 136

Screening 336

Serial amniocenteses 15

Single-needle insertion 221

Spontaneous abortion 336, 340

Stillbirth 248

Supraventricular tachycardia 328

Surgery 7

Tachycardia 205 Teratogen 292 Thyroid 24 Tissue ischemia 356 Total anomalous pulmonary venous connection 266

Transvaginal color Doppler 178

- $\quad$ ultrasound 132,336

TRAP sequence 50 
Tricuspid valve regurgitation 43

Triplets 59

Trisomy 18255,270

21210,270

screening 93 Tumor necrosis factor- $\alpha 7$ Twin-twin transfusion syndrome 15, 50 Twins 153,301

Ultrasonography 72, 156, 197 Ultrasound 153, 185, 286, 343, 363 Umbilical arterial blood

pressure 149

cord compromise 81

vein thrombosis 50

venous pressure 348 Ureaplasma urealyticum 89 Urinary ß-core fragment 340 Uteroplacental insufficiency 163 Uterus didelphys 59

Variability 170

Vascular disruption birth defects 292

Vibratory acoustic stimulation 167

Walker-Warburg syndrome 197

$\mathrm{X}$-linked ichthyosis 36

Fetal Diagn Ther Vol. 12, 1997

Subject Index 